\title{
SEPARATION AND SLIP BETWEEN A LAYER AND A SUBSTRATE CAUSED BY A TENSILE LOAD
}

\author{
D. SCHMUESER* and MARIA COMNINOU \\ The University of Michigan, Ann Arbor, MI 48109, U.S.A. \\ and \\ J. DUNDURS \\ Northwestern University, Evanston, IL 60201, U.S.A. \\ (Communicated by I. N. SNEDDON)
}

\begin{abstract}
The paper treats the elastic layer that is pressed uniformly against a half space and is subsequently subjected to a concentrated force tending to induce slip and separation between the bodies. The formulation, based on known results for discrete dislocations, reduces the problem to a coupled system of integral equations which is solved numerically. Various results of interest are given graphically.
\end{abstract}

\section{INTRODUCTION}

SUPPOSE THAT an elastic layer is pressed uniformly against a substrate and subsequently subjected to a concentrated load tending to separate locally the two bodies. It was shown in a previous paper [1] that, under these circumstances, slip takes place before separation is reached for any value of the friction coefficient. In the present article we pursue the separation phase of the loading process, after the slip zones have merged and a gap develops. For the sake of brevity, we omit many definitions of symbols and rely on several results given before[1].

\section{FORMULATION}

The geometry of the layer and the substrate with the anticipated separation and slip zones is shown in Fig. 1. The separation zone extends from $-b$ to $b$, and the slip zones cover the intervals $(-c,-b)$ and $(b, c)$. The layer and the substrate, the latter modeled as an elastic half space, are supposed to be made of the same material.

The solution of the unilateral problem involving separation and slip is constructed by superposing on the bilateral solution[1] corrective fields designed to satisfy the boundary conditions in the separation and slip zones. The boundary conditions in the slip and stick zones are as before [1]. The boundary conditions in the separation zone $|x|<b$ are

$$
N(x)=0, \quad S(x)=0, \quad g(x)>0
$$

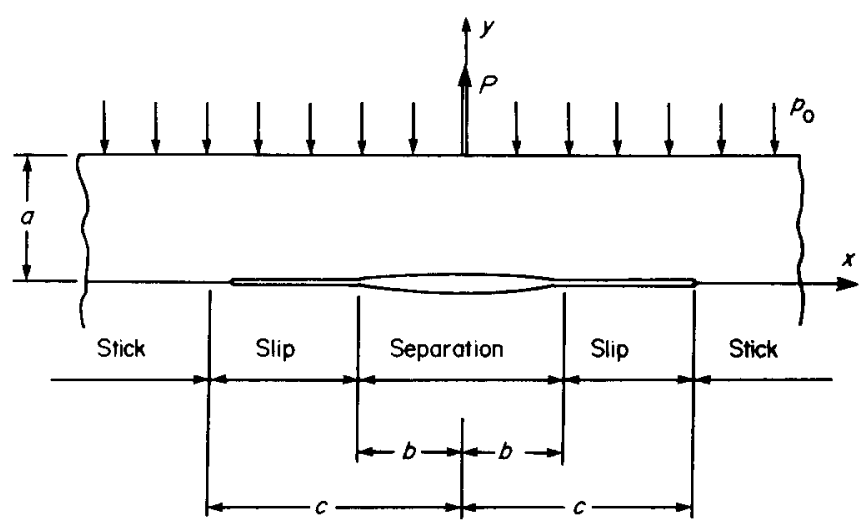

Fig. 1. Separation and slip zones.

${ }^{*}$ Currently at General Motors Research Laboratories, Warren, Michigan, U.S.A. 
where $g(x)$ denotes the gap developing between the solids, or

$$
g(x)=u_{y}^{(1)}(x, 0)-u_{y}^{(2)}(x, 0) .
$$

In order to construct the corrective solution we cover the interval $(-c, c)$ with a distribution $B_{x}(x)$ of glide dislocations and the interval $(-b, b)$ with climb dislocations of density $B_{y}(x)$. The tractions on $y=0$ for a discrete dislocation situated at $x=\xi, y=0$ were given in [1]. The tractions induced by a discrete climb dislocation are [2-4]

$$
\begin{aligned}
\tau_{x y}(x, 0)= & \frac{16 \mu b_{y}}{\pi(\kappa+1)}\left\{\frac{3 a^{3}}{\left[4 a^{2}+(x-\xi)^{2}\right]^{2}}-\frac{16 a^{5}}{\left[4 a^{2}+(x-\xi)^{2}\right]^{3}}\right\} \\
\tau_{y y}(x, 0)= & \frac{2 \mu b_{y}}{\pi(\kappa+1)}\left\{\frac{1}{x-\xi}-\frac{x-\xi}{4 a^{2}+(x-\xi)^{2}}-\frac{4 a^{2}(x-\xi)}{\left[4 a^{2}+(x-\xi)^{2}\right]^{2}}\right. \\
& \left.-\frac{128 a^{4}(x-\xi)}{\left[4 a^{2}+(x-\xi)^{2}\right]^{3}}\right\} .
\end{aligned}
$$

Using (6) above and eqn (11) of [1], (1) becomes

$$
\begin{aligned}
\frac{\pi(\kappa+1)}{2 \mu} N(x)= & -\int_{-b}^{b} \frac{B_{y}(\xi)}{\xi-x} \mathrm{~d} \xi-\int_{-b}^{b} B_{y}(\xi) k_{3}(x, \xi) \mathrm{d} \xi \\
& -\int_{-c}^{c} B_{x}(\xi) k_{4}(x, \xi) \mathrm{d} \xi+\frac{\pi(\kappa+1)}{2 \mu} \\
& \times\left\{-p_{0}+\frac{2 P}{\pi a} \frac{a^{4}}{\left(a^{2}+x^{2}\right)^{2}}\right\}=0, \quad|x|<b
\end{aligned}
$$

where

$$
\begin{aligned}
& k_{3}(x, \xi)=\frac{x-\xi}{4 a^{2}+(x-\xi)^{2}}+\frac{4 a^{2}(x-\xi)}{\left[4 a^{2}+(x-\xi)^{2}\right]^{2}}+\frac{128 a^{4}(x-\xi)}{\left[4 a^{2}+(x-\xi)^{2}\right]^{3}} \\
& k_{4}(x, \xi)=\frac{24 a^{3}}{\left[4 a^{2}+(x-\xi)^{2}\right]^{2}}-\frac{128 a^{5}}{\left[4 a^{2}+(x-\xi)^{2}\right]^{3}} .
\end{aligned}
$$

Anticipating the direction of slip for a steadily increased magnitude of the applied concentrated force $P$, the relation between the shearing and normal tractions is

$$
S(x)=f \operatorname{sgn} x N(x), \quad b<|x|<c .
$$

Because of (1) and (2), this relation can be rewritten as

$$
S(x)=f \operatorname{sgn} x N(x), \quad 0<|x|<c .
$$

Using (5) and (7) above, and eqn (11) of [1], this condition becomes

$$
\begin{aligned}
\int_{-c}^{c} \frac{B_{x}(\xi)}{\xi-x} \mathrm{~d} \xi & +\int_{-c}^{c} B_{x}(\xi) k_{5}(x, \xi) \mathrm{d} \xi+\int_{-b}^{b} B_{y}(\xi) k_{6}(x, \xi) \mathrm{d} \xi \\
& =-\frac{\pi(\kappa+1)}{2 \mu}\left\{\frac{2 P}{\pi a} \frac{a^{3} x}{\left(a^{2}+x^{2}\right)^{2}}+f \operatorname{sgn} x N(x)[H(|x|-b)-H(|x|-c)]\right\}, \quad|x|<c
\end{aligned}
$$

where

$$
\begin{aligned}
& k_{5}(x, \xi)=\frac{x-\xi}{4 a^{2}+(x-\xi)^{2}}-\frac{12 a^{2}(x-\xi)}{\left[4 a^{2}+(x-\xi)^{2}\right]^{2}}+\frac{64 a^{4}(x-\xi)}{\left[4 a^{2}+(x-\xi)^{2}\right]^{3}} \\
& k_{6}(x, \xi)=-k_{4}(x, \xi) .
\end{aligned}
$$


$N(x)$ is given by the expression entering (7), and $H()$ is the Heaviside step function.

Equations (7) and (13) form a coupled system of singular integral equations with generalized Cauchy kernels, subject to the conditions

$$
\int_{-c}^{c} B_{x}(\xi) \mathrm{d} \xi=0, \quad \int_{-b}^{b} B_{y}(\xi) \mathrm{d} \xi=0
$$

In addition, we note that

$$
B_{x}(x)=B_{x}(-x), \quad B_{y}(x)=-B_{y}(-x)
$$

which are implied by the symmetry of the problem. Hence, (16) is satisfied automatically. However, the numerical technique to be used for solving the integral equations does not incorporate these properties of the dislocation densities, and (17) and (18) must be verified after the solution is obtained. It must also be noted that both unknown functions $B_{x}(x)$ and $B_{y}(x)$ are bounded at the end points of the slip and separation zones, respectively [5].

\section{NUMERICAL SOLUTION AND RESULTS}

For the numerical solution, we first normalize the intervals $(-b, b)$ and $(-c, c)$ in $(7)$ and (12) by the change of variables

$$
\begin{array}{ll}
r=\xi / b, & s=x / b \\
\hat{r}=\xi / c, & \hat{s}=x / c .
\end{array}
$$

Then we set

$$
\begin{aligned}
& B_{x}(\hat{r})=\frac{P(\kappa+1)}{2 \mu a}\left(1-P^{2}\right)^{-1 / 2} \Phi_{x}(\hat{r}) \\
& B_{y}(r)=\frac{P(\kappa+1)}{2 \mu a}\left(1-r^{2}\right)^{1 / 2} \Phi_{y}(r)
\end{aligned}
$$

The density $B_{x}(\hat{f})$ given by (23) is assumed singular for the numerical computations only, and we cancel the singularity by requiring that

$$
\Phi_{x}(1)=\Phi(-1)=0 .
$$

We discretize the singular integral in (7) by the Erdogan-Gupta quadrature [6], the singular integral in (12) by the Theocaris-Ioakimidis quadrature [7], and the remaining regular integrals by the Gauss-Chebyshev quadrature [8]. Thus (7) and (12) become, respectively,

$$
\begin{aligned}
& \sum_{k=1}^{n}\left\{\frac{1-r_{k}^{2}}{n+1}\left[\frac{\Phi_{y}\left(r_{k}\right)}{r_{k}-s_{j}}+b \Phi_{y}\left(r_{k}\right) k_{3}\left(r_{k}, s_{j}\right)\right]+c W_{k} \Phi_{x}\left(\hat{r}_{k}\right) k_{4}\left(\hat{r}_{k}, s_{j}\right)\right\} \\
& =-\frac{p_{0} a}{P}+\frac{2 a^{4} / b^{4}}{\pi\left(a^{2} / b^{2}+s_{j}^{2}\right)^{2}}, \quad j=1,2, \ldots, n+1 \\
& \sum_{k=1}^{n}\left\{W_{k}\left[\frac{\Phi_{x}\left(\hat{r}_{k}\right)}{\hat{r}_{k}-\hat{s}_{i}}+c \Phi_{x}\left(\hat{r}_{k}\right) k_{5}\left(\hat{r}_{k}, \hat{s}_{i}\right)\right]+\frac{1-r_{k}^{2}}{n+1} \Phi_{y}\left(r_{k}\right) k_{6}\left(r_{k}, \hat{s}_{i}\right)\right\} \\
& =-\frac{2}{\pi} \frac{\left(a^{3} / c^{3}\right) \hat{s}_{i}}{\left(a^{2} / c^{2}+\hat{s}_{i}^{2}\right)^{2}}-f \operatorname{sgn} s_{i} N\left(s_{i}\right)\left[H\left(\left|\hat{s}_{i}\right|-\frac{b}{c}\right)-H\left(\left|\hat{s}_{i}\right|-1\right)\right], \\
& i=1,2, \ldots, n-1
\end{aligned}
$$


where

$$
\begin{array}{ll}
r_{k}=\cos \frac{k \pi}{n+1}, & k=1,2, \ldots, n \\
s_{i}=\cos \frac{(2 j-1) \pi}{2(n+1)}, & j=1,2, \ldots, n+1 \\
\hat{r}_{k}=\cos \frac{(k-1) \pi}{n-1}, & k=1,2, \ldots, n \\
\hat{s}_{i}=\cos \frac{(2 i-1) \pi}{2(n-1)}, & i=1,2, \ldots, n-1
\end{array}
$$

and

$$
\begin{gathered}
W_{1}=W_{n}=\frac{1}{2(n-1)} \\
W_{k}=\frac{1}{n-1}, \quad k=2, \ldots, n-1 .
\end{gathered}
$$

The discretized form of (15) is

$$
\sum_{k=1}^{n} W_{k} \Phi_{x}\left(\hat{r}_{k}\right)=0
$$

It may also be noted that the system of equations in (26) incorporates the consistency condition required for a bounded solution. In all, we have $2 n+2$ equations from (25)-(27) and (34) for the $2 n+2$ unknowns $\Phi_{x}\left(\hat{r}_{k}\right), \Phi_{y}\left(r_{k}\right), b$ and $c$. Equation (16) and one of (25) are not used because of symmetry which is found to be automatically satisfied.

The unknown parameters $b$ and $c$ defining the extents of the slip and separation zones make the algebraic system highly nonlinear. To simplify the iteration procedure, we specify $b / a$, and leave $c / a$ and the loading parameter $\lambda=P / p_{0} a$ as unknowns. Choosing $n$ as even, we use the $n / 2+1$ equation of system (26) to solve for $\lambda$ and to eliminate it from the remaining equations. Moreover, by using the Theocaris-Ioakimidis scheme, we can directly incorporate (25) into the algebraic matrix and eliminate one unknown at the same time, because the end points belong to the collocation points defined by (30). Thus we are left with a system of $2 n$ equations ( $n$ equations from (26), $n-1$ equations from (27) and one equation from (34)) for $2 n$ unknowns ( $n$ values of $\Phi_{y}\left(r_{k}\right), n-1$ values of $\Phi_{x}\left(\hat{r}_{k}\right)$ and the unknown $\left.c / a\right)$. For fixed $b / a$, we choose a value for $c / a$ and solve the system (26) with $j=1,2, \ldots n / 2, n / 2+2, \ldots, n+1$ and (27) for $\Phi_{x}\left(\hat{r}_{k}\right)$ and

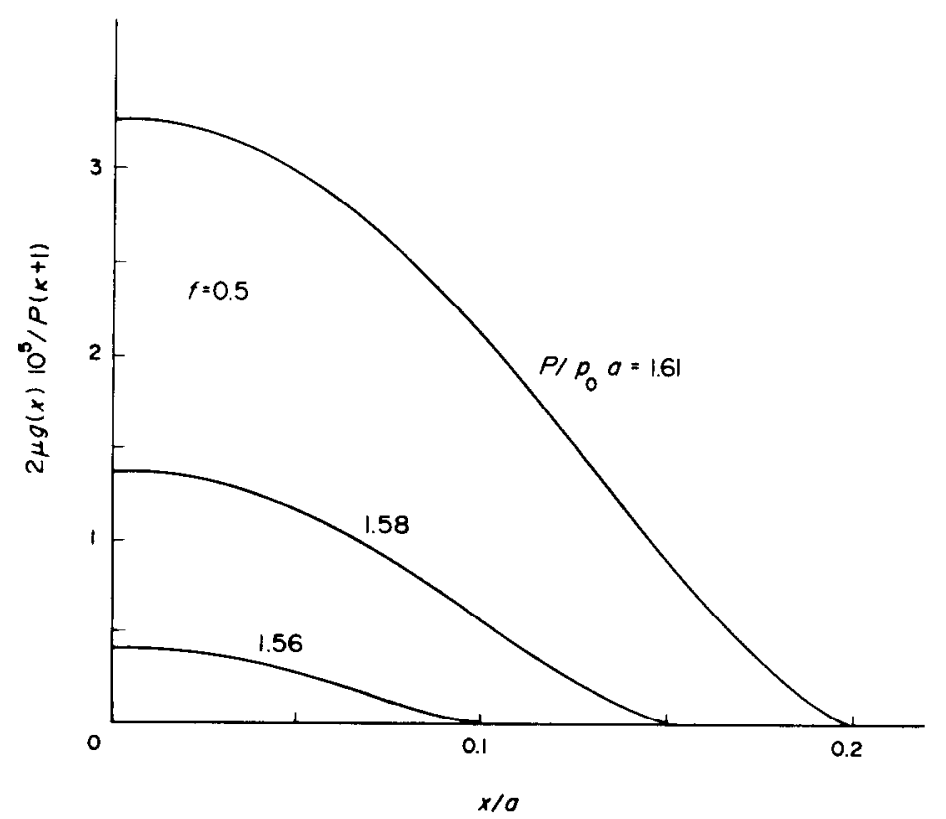

Fig. 2. Gaps for $f=0.5$ and different values of $\lambda$. 
$\Phi_{y}\left(r_{k}\right)$. Then we substitute into (34) which is generally not satisfied. We proceed by iteration until a value of $c / a$ is found so that (34) is satisfied. For this value, we verify that (16)-(18) are satisfied and also check the various inequalities for the problem [1].

The results thus obtained are shown in Figs. 2-8. Figure 2 shows the gaps for different values of $\lambda$ and $f=0.5$. Figure 3 shows the tangential shift $h(x)[1]$ in the interval $(0, c)$ for $f=0.5$ and different values of loading parameter $\lambda$. The effect of friction on $h(x)$ is depicted in Fig. 4 for the fixed value $b / a=0.4$. Figure 5 shows the effect of friction on the normal tractions and Fig. 6 the effect on the shearing tractions for $b / a=0.4$. The variation of $c / a$ with $\lambda$ for various values of the friction coefficient is given in Fig. 7, and that for $b / a$ in Fig. 8.

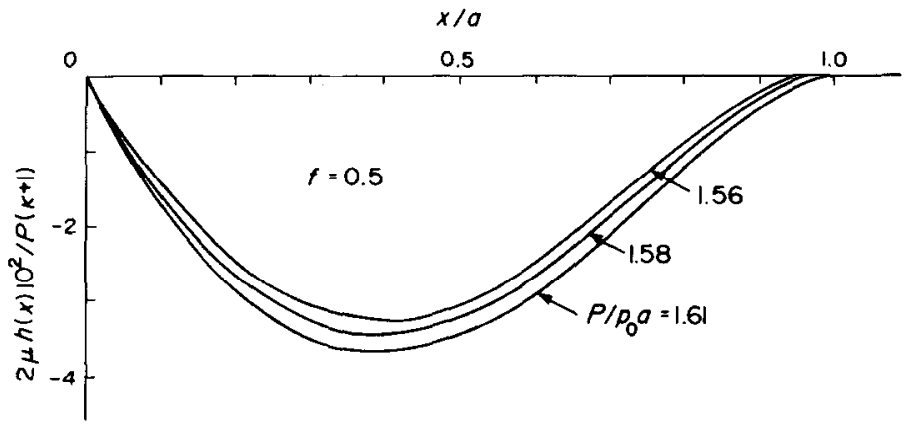

Fig. 3. Tangential shifts for $f=0.5$ and different values of $\lambda$.

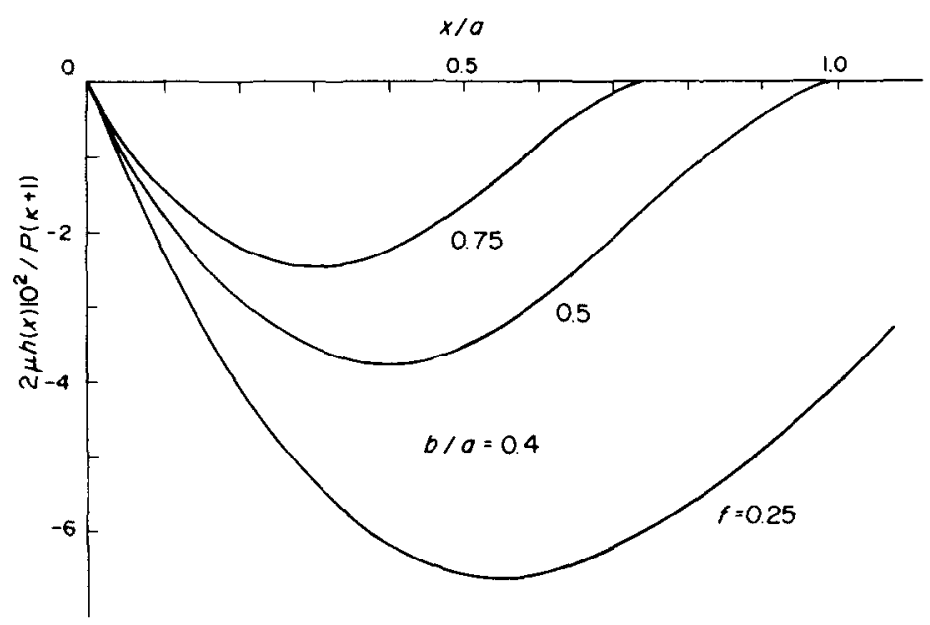

Fig. 4. Tangential shift for $b / a=0.4$.

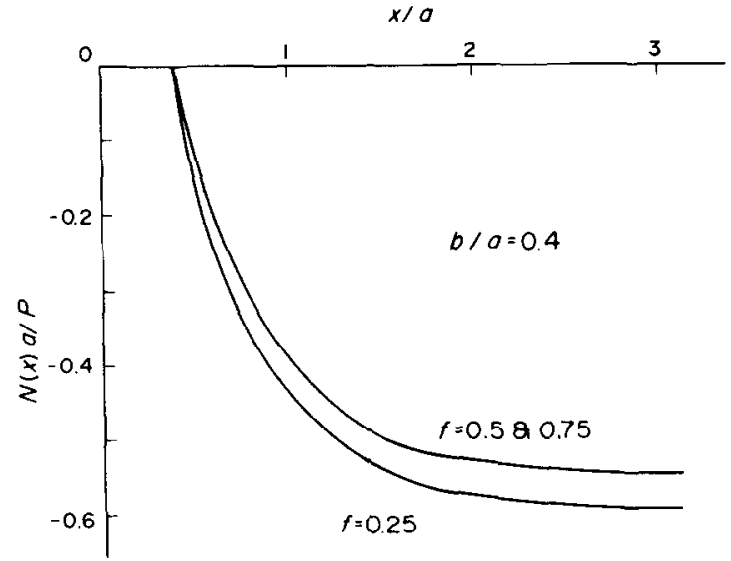

Fig. 5. Normal tractions for $b / a=0.4$. 


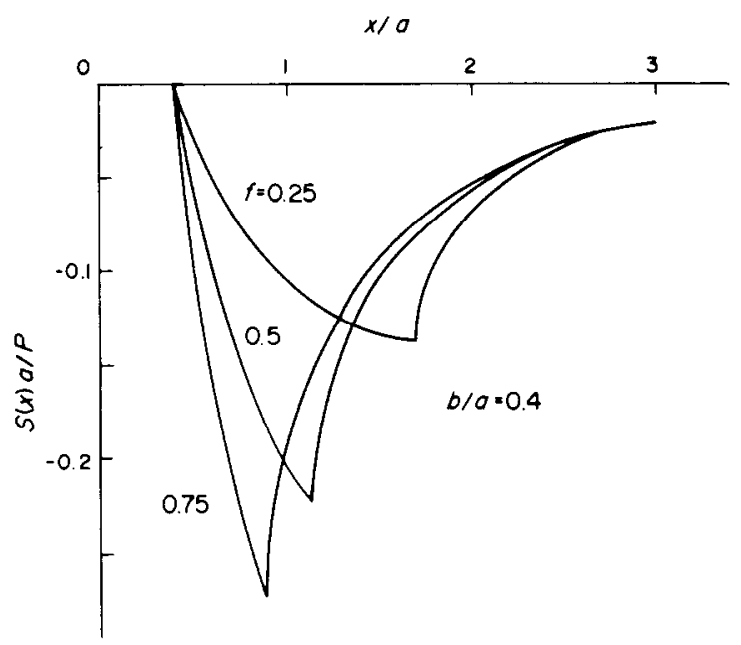

Fig. 6. Shearing tractions for $b / a=0.4$.

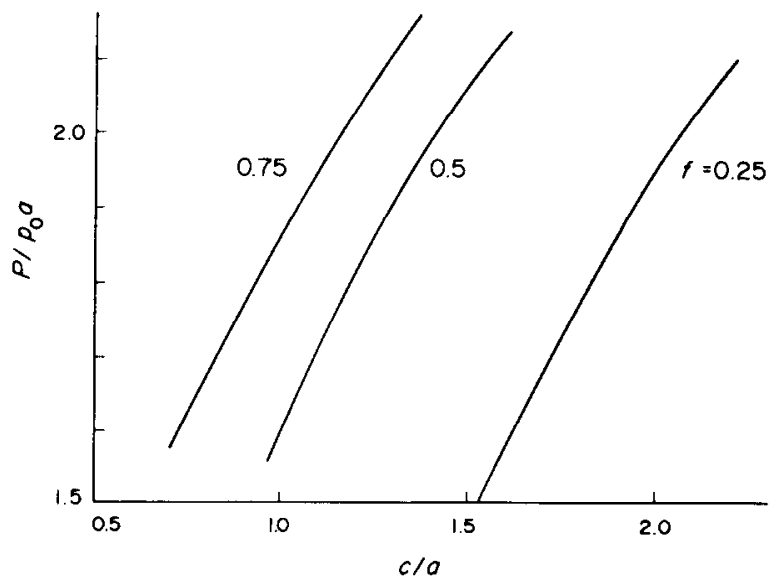

Fig. 7. Variation of $c / a$ with $\lambda$.

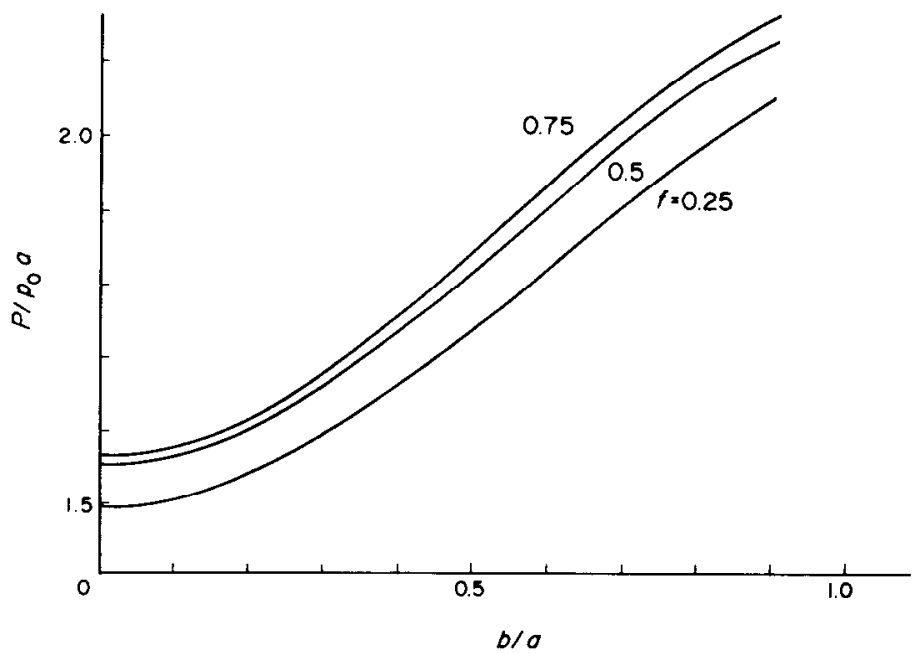

Fig. 8. Variation of $b / a$ with $\lambda$. 
Acknowledgement - One of the authors (M.C.) wishes to acknowledge the support by the National Science Foundation under the grant ENG 77-25302.

\section{REFERENCES}

[1] M. COMNINOU, D. SCHMUESER and J. DUNDURS, Int. J. Engng Sci. 18, 131 (1980).

[2] J. DUNDURS and T. MURA, J. Mech. Phys. Solids 12, 177 (1964).

[3] J. DUNDURS and G. P. SENDECKYJ, J. Appl. Phys. 36, 3353 (1965).

[4] J. DUNDURS, In Mathematical Theory of Dislocations (Edited by T. Mura), pp. 70-115. American Society of Mechanical Engineers (1969)

[5] J. DUNDURS and M. COMNINOU, J. Elasticity 9, 71 (1979).

[6] F. ERDOGAN and G. GUPTA, Q. Appl. Math. 29, 525 (1972).

[7] P. S. THEOCARIS and N. I. IOAKIMIDIS, Q. Appl. Math. 35, 173 (1977).

[8] A. H. STROUD and D. SECREST, Gaussian Quadrature Formulas. Prentice-Hall, Englewood Cliffs, New Jersey (1966).

(Received 9 October 1979) 